\title{
ENVEJECIMIENTO MASCULINO EN LA EDAD MEDIA: COMENTARIOS AL LIBRO DE ARNAU DE VILANOVA "CONSERVATIO JUVENTUTE PRESERVATIO SENECTUTE
}

\author{
Remigio Vela Navarrete y Mariano Pérez Albacete?. \\ Fundación Jiménez Díaz. Madrid y Hospital Universitario Virgen de la Arrixaca'. Murcia. España.
}

\begin{abstract}
Resumen.- En los textos médicos de la Edad Media las notas sobre las medidas de higiene personal son escasas y se encuentran dedicadas a los reyes o a la aristocracia. En su obra "Regimen sanitatis ad inclytum regen Aragorum", Arnaldo de Vilanova subraya seis cosas necesarias para el aseo, entre las que se incluye su famosa proposición "Conservatio juventute preservatio senectute". Hemos comparado el texto al de otros autores con referencias al tema y, una vez examinados, destaca el escrito por Vilanova. Los médicos en la Edad Media se encontraban ya interesados en el envejecimiento y propuestas consideradas hoy día como modernas las encontramos descritas en diversas obras de la época.
\end{abstract}

Palabras clave: Renacimiento. Arnaldo de Vilanova. Higiene en la Edad Media. Historia de la Urología.

Summary.- In the Middle Ages medical texts the notes about personal hygiene are scarce and they are dedicated to the Kings or the aristocracy. In his work Regimen sanitatis ad inclytum regen Aragorum, Arnaldo de Vilanova underlines six things that are necessary for cleanliness, among which he includes his well-known proposition Conservatio juventute preservatio senectute. We compared his text with those from other authors with references to the issue and, once examined, Vilanova' $s$ work stands out. Doctors in the middle ages were already interested in aging and propositions considered today as modern are found described in various works from that period.

Keywords: Renaissance. Arnaldo de Vilanova. Hygiene in the Middle Ages. History of Urology.

\section{INTRODUCCIÓN}

Los textos relacionados con el cuidado personal en la Edad Media se refieren fundamentalmente al varón y están dedicados a los reyes o a la aristocracia. Recogen recomendaciones hipocráticas arabizadas y latinizadas más tardíamente al comenzar el Renacimiento. Destaca entre estos, el escrito por Arnau de Villanova, Conservatio juventute preservatio senectute, cuyo título es sorprendentemente moderno y provocativo. 


\section{RESULTADO}

Arnaldo de Vilanova (Figura 1) es una de las máximas figuras de la Europa bajo medieval, momento en que conviven la medicina de Bizancio, el galenismo arabizado de Bagdad y de Al-Andalus y las escuelas de Salerno y de Montpellier. Arnaldo de Vilanova (ca. 1238-1311), cristiano, descendiente de una familia que se instaló en Valencia inmediatamente después de su conquista por Jaime I, estudió Medicina en Montpellier (1260) y ejerció en Valencia y en Barcelona como médico de cámara de los reyes de Aragón Pedro el Grande, Alfonso III y Jaime II. En 1290 y durante nueve años fue profesor en la Universidad de Montpellier, donde elevó el nivel de la enseñanza, con especial entusiasmo por Galeno y donde redactó la mayor parte de su obra médica, considerada como paradigma del galenismo arabizado. Combatió el pragmatismo escolástico de la época atribuyéndolo de escasa ayuda para la práctica médica, especialmente en sus especulaciones ya que el médico solamente conoce mediante lo que se

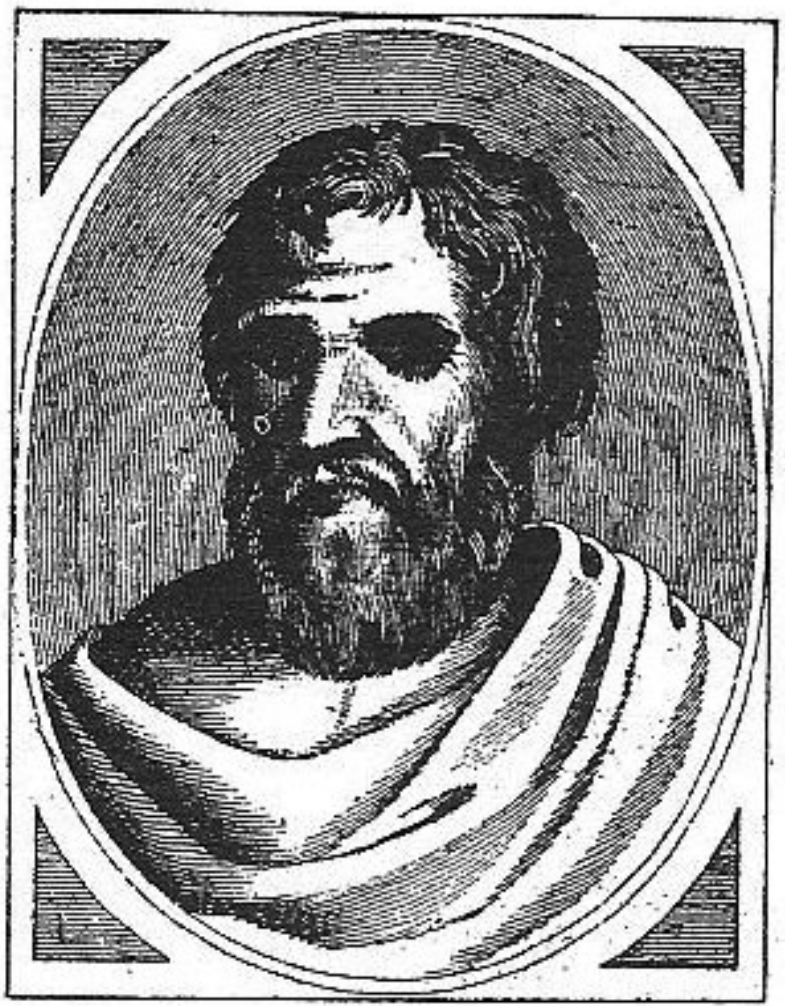

\section{A R N A L D V S Villanouanus.}

manifiesta a los sentidos. Defendió la importancia de la observación clínica, como Rhazes y combatió agriamente a Averroes, está reputado como un precursor del Renacimiento. Su producción médica fue extensísima, sobrepasa los cincuenta y cinco libros (Figura 2), entre ellos destaca su Régimen sanitatis ad inclytum regem aragonum Jaume II (Régimen de salud para el ínclito rey de Aragón Jaime III), en el que divulga medidas higiénicas y normas para la atención y conservación de la salud que persiguen demostrar que, mediante cuidados y un régimen sano, se puede disfrutar de la vida y se puede llegar a natural vellea (a viejo de natural).

Su obra tuvo una amplia difusión en toda Europa, pero se incluyó su nombre en libros sobre magia y alquimia, por su fama y prestigio, que no fueron escritos por el.

Tanto el texto previamente señalado como su Speculun Medicinae coincidió con la publicación en Europa de otras obras sobre la higiene personal y la
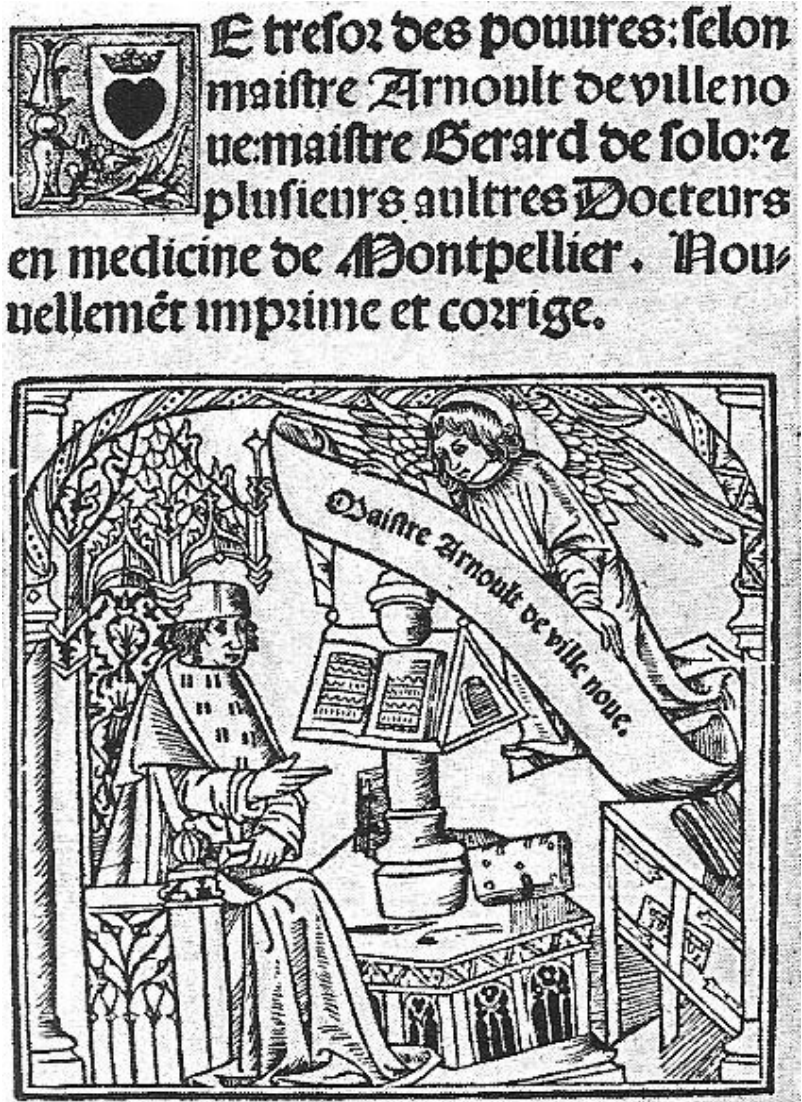

TOn les vend a Zport en la moifonse Llaudc Houtry dict le hotucc/au pics neftre oame oc esonfort.

FIGURA 2. Grabado de madera de tesoro de los 
TABLA I. REGIMINA SANITATIS EN TEXTOS GRECO LATINOS Y BIZANTINOS.

\begin{tabular}{|l|}
\hline HIPOCRATES: ACERCA DE LA DIETA \\
\hline DIOCLES DE CARYSTUS (320-3 10 a. C.): CARTA A LOS ANTÍGONOS \\
\hline $\begin{array}{c}\text { GALENO: DE SANITATITE TUENDA (180 d. C.) } \\
\text { DE ALIMENTORUM FACULTATIBUS }\end{array}$ \\
$\begin{array}{c}\text { AMTHIMO: DE OBSERVATIONE CIBORUM (V-VI) (Epistula anthimi comitis et legendarii ad gloriossimus } \\
\text { theodoricum regem francorum) }\end{array}$ \\
\hline
\end{tabular}

salud pública como el Líber de conservanda sanitate, de Juan de Toledo, de quien sabemos que falleció 1275 y por lo tanto anterior a la publicación del aragonés. Pero el prestigio de la obra de este último es tal que hasta en el 1606 Jerónimo de Mondragón hace una traducción al castellano del original en latín. Entre los libros leídos sobre estos temas en aquella época, especialmente para ricos y nobles, hay que citar también la traducción al latín del de Ibn Butlan o Tablas de salud sobre las seis cosas que son necesarias, tablas ricas en dibujos, lujosamente ilustradas, de notable difusión a finales de la Edad Media y que tendrían su continuidad con otros muy clásicos dedicados a la higiene personal en la España renacentista (Figuras 3 y 4 ).

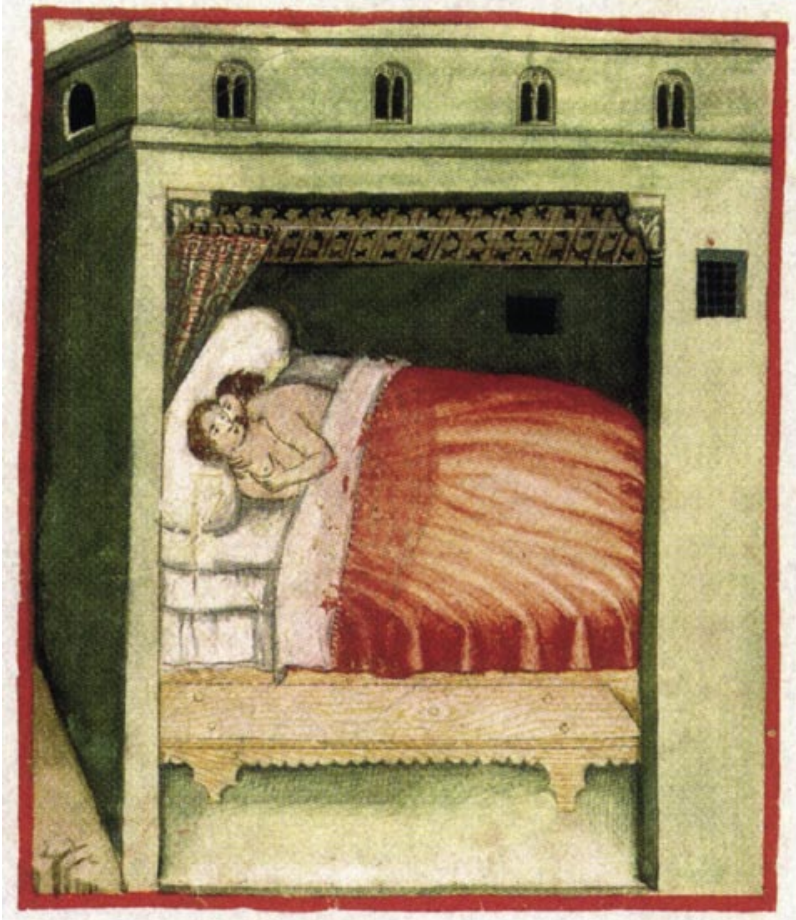

El contenido de estas obras, en general, sigue un orden concreto en el que se refiere a los mejores lugares para conservar la salud y alargar la vida, normas sobre el ejercicio del baño y de la higiene personal, del comer y del beber, del dormir y el velar, de la actividad sexual, de las pasiones del ánimo y presenta también, un extenso capítulo dedicado a la alimentación y a la bebida. Estos libros tienen su continuidad en el renacimiento castellano y hay que citar los siguientes: Banquete de nobles caballeros (Figura 5), editado el 1530 por Luis Lobera de Ávila, en el que observamos una intención individualizada y personal ya que lo redactó cuando Carlos $V$ acababa de nombrarlo su médico de cámara, tras asistir a la coronación imperial en Bolonia, ciudad donde

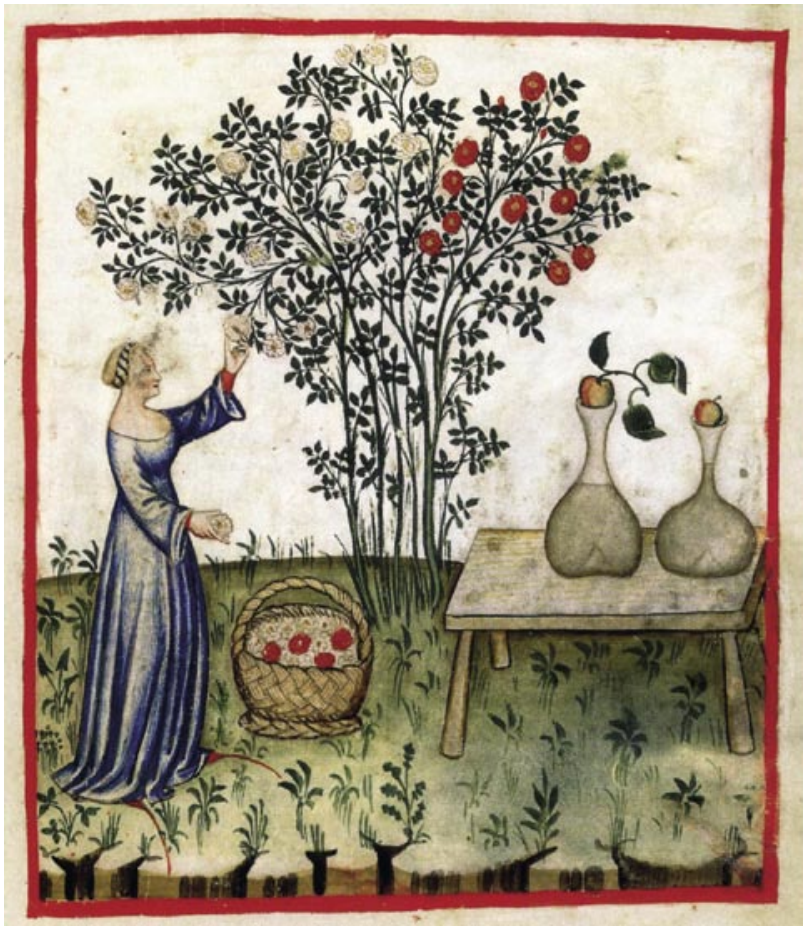

FIGURAS 3 Y 4 . Dibujos de las Tablas de la Salud de Ibn Burlan. 


\section{TABLA II. REGIMINA SANITATIS EN LA MEDICINA ÁRABE Y EN LA EDAD MEDIA (Traducidos en Toledo, Roma y Montpelier)}

\begin{tabular}{|l|}
\hline ISAAC IUDAEUS (Isaac Israili) (s. IX): LIBER DIETARUM UNIVERSALIUM PARTICULARIUM \\
\hline JOHANNITIUS (Hunain Ibn Ishaq) (s. X): ISAGOGE \\
\hline ALI ABBAS (Ali ibn al-Abbas al Magusi) (s. X): PANTEGNI \\
\hline AVICENA/IBN SINA (980-1037): CANON (Toledo, 1157) \\
\hline RAZES (Abubark Muhammad Ibn Zakariyya Ar-Razi) (865-902): LIBER AL ADMANSOREM \\
\hline AVERROES (Ibn Rusd) (1 126-1 198): COLLIGET (KITAB AL-KULLIYAT) \\
\hline AVENZOAR (Abul/Ala Ibn Zhur): TAISIR REGIMINIA SANITATIS (1299) \\
\hline MAIMONIDES. (E. Blasi, 1302) \\
\hline BERNARDO DE GORDONIO: DE CONSERVATIONE VITAE HUMANAE (editado en 1303) \\
\hline ARNAU DE VILLANOVA (1240-1331): REGIMEN SANITATIS AD REGEM ARAGORUM (1305-1308) \\
\hline JUAN DE TOLEDO (s. XII): CONSERVANDA SANITATE (1224?) \\
\hline ALDOBRANDINO DE SIENA: REGIMINA SANITATIS (1234-1256) \\
\hline PETRIS HISPANUS (1205-1277): 4 Libros relacionados con REGIMINA SANITATIS \\
\hline MAINO DE MAINIERI: REGIMEN SANITATIS AD A. FLISCO 8 (1330) \\
\hline
\end{tabular}

\section{VANQVETEDE NOB"} LES CAVALLEROS E MODO DE BIVIR DESDE que feleuantan hafta đ̃ fe acueftan, y habla de Cada manjarque complexion y propiedad tienee que dañosy prouechos hase,etra ta deI regimiento curatiuo e preferuatiuo delas fiebres Peftilencia les e dela Peftilencia e otras cofas uriliftimes, nueuamente com: puefto porel Doctor Luysde Auila Medico defu Mageftad, diri gido af Illuftre y muy magnifico Señor el, S.don Francifco delos Couos Comendador mayor de Leon Secretario y del confejo del eftado y fecreto de fu Mageftad \&c.

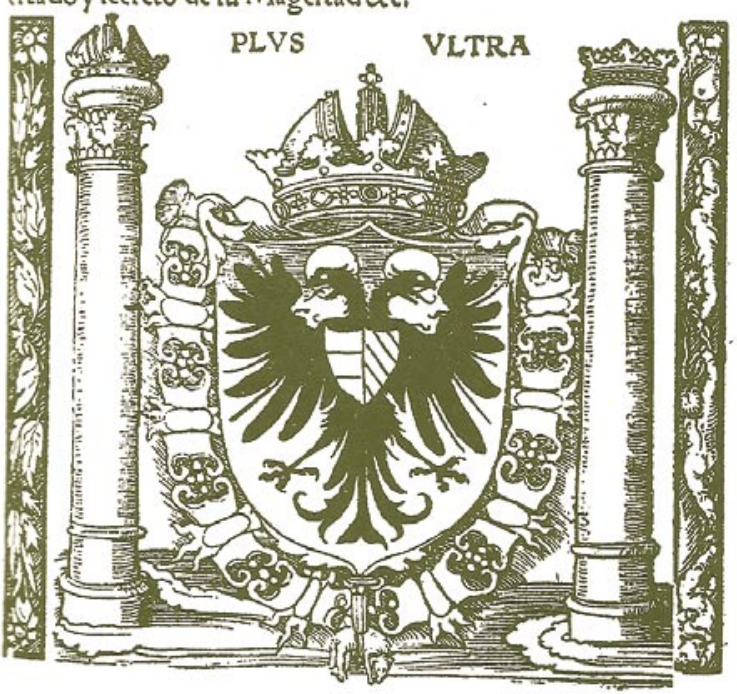

Conptiuilegio Imperıal,

FIGURA 5. Portada de la obra Banquete de Nobles Caballeros de Lobera de Ávila, 1530. fue impreso por primera vez. Forma parte de una colección de libros dedicados al llamado «regímina sanitatis»; a la misma línea corresponden varios tratados de la segunda mitad del siglo XVI, como el Libro intitulado la conservación de la salud del cuerpo y el alma, de Blas Álvarez de Miraval (1597) y el Aviso de Sanidad, de Francisco Núñez de Oria (1569) así como el Libro del ejercicio corporal y de sus provechos, de Cristóbal Méndez (1553).

\section{BIBLIOGRAFÍA Y LECTURAS RECOMENDADAS (*lectura de interés $y^{* *}$ lectura fundamental)}

**1. GARCÍA BALLESTER, L.: “Arnaldo de Vilanova Opera Medica Omnia". 11 volúmenes, Barcelona, 1996.

*2. GASCÓN VILLAPLANA, P.: "Estudio sobre Arnau de Vilanova". Medicina e Historia, (46) edt. Uriach Barcelona, 1975.

3. GRANJEL, L.S.: "El ejercicio médico y otros capítulos de la medicina española”. Ed. Universidad Salamanca, 1974.

4. LAÍN ENTRALGO, P.: "Historia de la Medicina”. Barcelona, Salvat, 1978.

5. LÓPEZ PIÑERO, J.M.: "Bibliografía América Hispánica". Valencia, 1987.

*6. PANIAGUA ARELLANO, J.A.: "La obra médica de Arnaldo de Vilanova”. Arch. Iberoamericano de Historia de la Medicina, XI: 351, Madrid, 1959. 